\title{
Beiträge zur Chemie des Bors, 153 [1] \\ Die Kristall- und Molekülstruktur eines 1.3.2-Dioxaborolan-2-yl-1.3.2-dioxaborolans
}

Contributions to the Chemistry of Boron, 153 [1]

The Crystal and Molecular Structure of a 1.3.2-Dioxaborolan-2-yl-1'.3'.2'-dioxaborolane

H. Nöth*

Institut für Anorganische Chemie der Universität München, Meiserstraße 1, D-8000 München 2

Z. Naturforsch. 39b, 1463-1466 (1984); eingegangen am 16. Juli 1984

Herrn Professor Dr. Dr. h. c. G. Fritz zum 65. Geburtstag gewidmet

X-Ray Structure

4.4.5.5-Tetramethyl-1.3.2-dioxaborolane-2-yl-4' $.4^{\prime} \cdot 5^{\prime} .5^{\prime}$-tetramethyl-1' $.3^{\prime} .2^{\prime}$-dioxaborolane

The structure of the title compound $\mathbf{5}$ has been investigated by X-ray crystallography in order to discern between two structural alternatives. $\mathbf{5}$ crystallizes in the monoclinic space group $\mathrm{P} 2{ }_{1} / c$, and almost planar $\mathrm{B}-\mathrm{B}$ bonded dioxaborolane rings are present in the molecule. $\mathbf{5}$ possesses a crystallographically imposed center of inversion, and approaches the point group symmetry $\mathrm{D}_{2 \mathrm{~h}}$.

Die Umsetzung von $\mathrm{B}_{2}\left(\mathrm{NMe}_{2}\right)_{4}\left(\mathrm{Me}=\mathrm{CH}_{3}\right)$ mit N.N'-Dimethylethylendiamin $[2,3]$ oder die Enthalogenierung von 2-Chlor-1.3-dimethyl-1.3.2-diazaborolidin [4] führen zu ein und derselben Verbindung, die röntgenstrukturanalytisch als das Diazaborolidin-2-yl-diazaborolidin (1) charakterisiert werden konnte [5]. Das Isomere $\mathbf{2}$ ist daher auszuschließen, obgleich massenspektrometrische Informationen mit Struktur $\mathbf{2}$ besser als mit $\mathbf{1}$ verträglich schienen [6]. Ein analoges Problem liegt auch bei den Produkten vor, die bei der Umsetzung von $\mathrm{B}_{2}\left(\mathrm{NMe}_{2}\right)_{4}$ mit Glykol $(3,4)$ oder Dithioglykolen in Gegenwart von $\mathrm{HCl}$ entstehen [7]. Die vorliegende Mitteilung beschreibt die durch Röntgenstrukturanalyse bestimmte Konfiguration von $\mathbf{5}$, das aus Tetrakis(dimethylamino)diboran(4) und Pinakol entsteht.

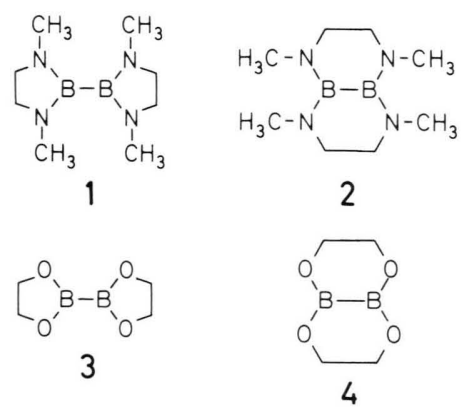

\footnotetext{
* Sonderdruckanforderungen an Prof. Dr. H. Nöth
} 0340-5087/84/1100-1463/\$ 01.00/0

\section{Kristall- und Molekülstruktur des}

\subsection{2-Dioxaborolan-2-yl-1' $.3^{\prime}$.2' -dioxaborolans 5}

5 wurde aus Pentan in farblosen, blättchenartigen Einkristallen erhalten. Ein Kristall $(0,18 \times 0,5 \times$ $0,7 \mathrm{~mm}$ ) wurde unter Argon in einer Glaskapillare montiert und am automatisierten Vierkreisdiffraktometer (Syntex P3) justiert; dann wurden zunächst 10 Reflexe zentriert. Zur Bestimmung der Zellparameter dienten die Winkelpositionen von 21 automatisch zentrierten Reflexen mit $19,9^{\circ}<2 \theta<31^{\circ}$. Die relevanten kristallographischen Daten einerseits sowie die der Datensammlung andererseits enthält Tab. I. Aus den systematischen Auslöschungen folgt die Raumgruppe $\mathrm{P} 2{ }_{1} / \mathrm{c}(h 0 l$ mit $l=2 n, 0 k 0$ mit $k=2 n)$. Die E-Statistik entsprach der zentrosymmetrischen Raumgruppe.

Die Strukturlösung erfolgte nach Datenreduktion ohne Absorptionskorrektur mit direkten Methoden (SHELXTL-Programmpaket). Die beste E-Map wies die Lagen aller Nichtwasserstoff-Atome auf. Nach Verfeinerung mit isotropen und danach mit anisotropen Temperaturfaktoren waren der Differenz-Fourier-Synthese die Lagen aller $\mathrm{H}$-Atome zu entnehmen. Wegen der relativ großen $\mathrm{U}_{\mathrm{ij}}$-Werte der Methyl-C-Atome führten wir die weitere Verfeinerung mit starren $\mathrm{CH}_{3}$-Gruppen sowie fixierten $\mathrm{CH}$ Abständen und $\mathrm{U}_{\mathrm{i}}$-Werten bis zur Konvergenz bei $R=0,065$ fort. Tab. II enthält die Ortskoordinaten und $\mathrm{U}_{\mathrm{ij}}$-Werte der anisotropen Temperaturparameter für die Nichtwasserstoff-Atome [8].

Die Röntgenstrukturanalyse lehrt, daß die untersuchte Diboran(4)-Verbindung ein Dioxaborolan-2- 
Tab. I. Kristallographische Daten von 5 sowie Angaben zur Datensammlung und Strukturlösung ( $\mathrm{MoK}_{\alpha}$-Strahlung, graphit-monochromatisiert, $\omega$-Scan).

\begin{tabular}{lc}
\hline Summenformel & $\mathrm{C}_{12} \mathrm{H}_{24} \mathrm{~B}_{2} \mathrm{O}_{4}$ \\
Molmasse & 253,93 \\
$a(\AA)$ & $10,423(8)$ \\
$b(\AA)$ & $7,625(9)$ \\
$c(\AA)$ & $10,334(2)$ \\
$\beta\left({ }^{\circ}\right)$ & $110,81(12)$ \\
$\mathrm{V}\left(\AA^{3}\right)$ & $767,7(9)$ \\
$\mathrm{d}_{\mathrm{r}}\left(\mathrm{g}^{\mathrm{c}} \mathrm{cm}^{3}\right)$ & 1,10 \\
$Z$ & 2 \\
$\mathrm{Raumgruppe}$ & $\mathrm{P} 2{ }_{1} / \mathrm{c}$ \\
$\mu\left(\mathrm{cm}^{-1}\right)$ & 0,95 \\
$\mathrm{~F}(000)$ & 295,96 \\
Meßbereich 2 $\theta\left(^{\circ}\right)$ & $2-45$ \\
Meßgeschwindigkeit $\left({ }^{\circ} / \mathrm{min}\right)$ & $2,5-29,3$ \\
Meßbreite $\left({ }^{\circ}\right)$ & 1,2 \\
Gemessene Reflexe & $1221^{*}$ \\
davon Kontrollreflexe & 50 \\
Anzahl der symmetrie- & \\
unabhängigen Reflexe & 1073 \\
Anzahl der Reflexe mit I $>3 \sigma(\mathrm{I})$ & 788 \\
Anzahl der verfeinerten Parameter & 94 \\
$R$ & 0,0646 \\
$R_{\mathrm{w}}$ & 0,0662 \\
w & 0,0003 \\
\hline
\end{tabular}

* Davon war einer zu intensiv, ein weiterer zeigte zu starke Untergrundunterschiede.

yl-dioxaborolan (5) und nicht das isomere kondensierte 4-Analoge ist. Abb. 1 zeigt eine Abbildung der Molekülstruktur von $\mathbf{5}$ in ORTEP-Darstellung. Tab. III enthält ausgewählte Bindungsabstände und -winkel.

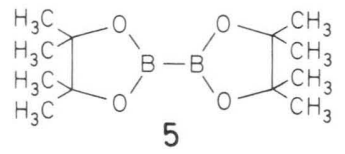

5 besitzt, kristallographisch bedingt, Zentrosymmetrie; das Inversionszentrum liegt im Mittelpunkt der B-B-Bindungsachse. Der Dioxaborolan-Ring ist nahezu planar (Abweichungen von der Ausgleichsebene: B 0,5, O1 1,0, O $20,3, \quad$ C $1-1,1$, C2 $0,8 \mathrm{pm})$. Dies hat zur Folge, daß die Methylgruppen nicht auf Lücke stehen; gleiches gilt auch für die $\mathrm{H}$-Atome (kürzester intramolekularer $\mathrm{H}-\mathrm{H}$-Kontakt zwischen Methylgruppe H6C-H5B 237,4 pm).

Im Vergleich mit den Ring-C-Atomen zeigen die Sauerstoff-Atome eine beachtliche SchwingungsAnisotropie: sie bewegen sich insbesondere senkrecht zur Ringebene. Dies legt nahe, daß die Dioxaborolan-Ringe in $\mathbf{5}$ tatsächlich nicht so eben sind, wie die Gleichgewichtslage nahelegt. Da die thermischen Ellipsoide der Ring-C-Atome im Gegensatz zu denen der Methyl-C-Atome nur eine geringe Anisotropie aufweisen, kann man den $\mathrm{U}_{\mathrm{ij}}$-Werten entnehmen, daß diese vor allem durch eine TorsionsSchwingung der Ring- $\mathrm{C}_{2} \mathrm{CO}$-Gruppen hervorgerufen werden. Konsequenterweise korrigiert eine Librationsanalyse vor allem die CC-Abstände. Die librationskorrigierten Werte finden sich in Spalte 3 der Tab. III.

Die Bor-Atome in $\mathbf{5}$ sind planar von den NachbarAtomen umgeben, d.h., wie zu erwarten, sind die BAtome als $\mathrm{sp}^{2}$-hybridisiert anzusehen. Allerdings liegt ein $\mathrm{O} 1-\mathrm{B}-\mathrm{O} 2-\mathrm{Winkel}$ vor, der mit $111,1^{\circ}$ kleiner als der Idealwinkel von $120^{\circ}$ ist. Die Bindungswinkel an den Sauerstoff-Atomen sind im Vergleich mit dem OBO-Winkel nur unwesentlich größer (im Durchschnitt $111,8^{\circ}$ ). Diese Bindungswinkel und jene an den Ring-C-Atomen (im Durchschnitt $102,7^{\circ}$ ) legen eine nicht unerhebliche Ringspannung im Dioxaborolan-System nahe.

Abb. 2 entnimmt man, daß im Gitter von $\mathbf{5}$ die Orientierung der Moleküle zueinander nicht durch $\mathrm{B} \cdots \mathrm{O}-$ Wechselwirkungen sondern durch $\mathrm{H} \cdots \mathrm{O}$ -

Tab. II. Atomkoordinaten $\left(\times 10^{4}\right)$ und Parameter des anisotropen Temperaturfaktorexponenten $\left(\AA^{2} \times 10^{3}\right)$ von 5. Der anisotrope Temperaturfaktorexponent ist definiert durch $-2 \pi\left(h^{2} a^{* 2} \mathrm{U}_{11}+\ldots 2 h k a^{*} b^{*} \mathrm{U}_{12}+\ldots\right)$. Standardabweichungen in Klammern.

\begin{tabular}{|c|c|c|c|c|c|c|c|c|c|}
\hline Atom & $\mathrm{U}_{11}$ & $\mathrm{U}_{22}$ & $\mathrm{U}_{33}$ & $\mathrm{U}_{12}$ & $\mathrm{U}_{13}$ & $\mathrm{U}_{23}$ & $x / a$ & $y / b$ & $z / c$ \\
\hline B & $41(2)$ & $51(2)$ & $45(2)$ & $-1(2)$ & $14(2)$ & $4(2)$ & $4353(3)$ & $10013(5)$ & $4209(3)$ \\
\hline $\mathrm{O}(1)$ & $74(2)$ & $138(3)$ & $62(2)$ & $46(2)$ & $-18(1)$ & $-53(2)$ & $3229(2)$ & $9043(4)$ & $3881(2)$ \\
\hline $\mathrm{O}(2)$ & $74(2)$ & $136(3)$ & $59(1)$ & $34(2)$ & $-12(1)$ & $-55(2)$ & $4337(2)$ & $11012(4)$ & $3157(2)$ \\
\hline$C(1)$ & $41(2)$ & $58(2)$ & $44(2)$ & $2(2)$ & $8(1)$ & $-1(2)$ & $2343(3)$ & $9321(4)$ & $2473(3)$ \\
\hline$C(2)$ & $46(2)$ & $57(2)$ & $40(2)$ & $4(2)$ & $8(1)$ & $1(2)$ & $3112(3)$ & $10748(4)$ & $1959(3)$ \\
\hline$C(3)$ & $72(3)$ & $94(3)$ & $147(4)$ & $-1(3)$ & $65(3)$ & $-3(2)$ & $975(4)$ & 9894(6) & $2534(5)$ \\
\hline$C(4)$ & $113(3)$ & $62(3)$ & $151(4)$ & $-28(3)$ & $67(3)$ & $-25(3)$ & $2179(5)$ & $7595(5)$ & $1725(5)$ \\
\hline$C(5)$ & $120(4)$ & $60(3)$ & $153(4)$ & 31(3) & $69(3)$ & $16(3)$ & $2415(5)$ & $12507(5)$ & $1628(5)$ \\
\hline$C(6)$ & $146(4)$ & $97(3)$ & $98(3)$ & 1(3) & $86(3)$ & $-6(3)$ & $3568(5)$ & $10201(6)$ & $783(4)$ \\
\hline
\end{tabular}


Tab. III. Bindungsabstände (in pm) und Bindungswinkel (in ${ }^{\circ}$ ) (Standardabweichungen in Klammern) sowie librationskorrigierte Werte.

\begin{tabular}{|c|c|c|c|}
\hline $\mathrm{B}-\mathrm{B}^{\prime}$ & $171,1(6)$ & 171,6 & \\
\hline $\mathrm{B}-\mathrm{O} 1$ & $132,4(4)$ & 132,8 & \\
\hline $\mathrm{B}-\mathrm{O} 2$ & $132,3(5)$ & 132,9 & \\
\hline $\mathrm{C} 1-\mathrm{O} 1$ & $143,5(3)$ & 144,2 & \\
\hline $\mathrm{C} 2-\mathrm{O} 2$ & $144,2(3)$ & 144,5 & \\
\hline $\mathrm{C} 1-\mathrm{C} 2$ & $155,3(5)$ & 156,2 & \\
\hline $\mathrm{C} 1-\mathrm{C} 3$ & $151,5(6)$ & 152,6 & \\
\hline $\mathrm{C} 1-\mathrm{C} 4$ & $150,5(5)$ & 151,3 & \\
\hline $\mathrm{C} 2-\mathrm{C} 5$ & $150,6(5)$ & 151,1 & \\
\hline $\mathrm{C} 2-\mathrm{C} 6$ & $151,2(6)$ & 151,7 & \\
\hline $\mathrm{O} 1-\mathrm{B}-\mathrm{O} 2$ & $111,1(2)$ & $\mathrm{C} 2-\mathrm{C} 1-\mathrm{C} 3$ & $114,8(3)$ \\
\hline $\mathrm{O} 1-\mathrm{B}-\mathrm{B}^{\prime}$ & $124,3(4)$ & $\mathrm{C} 2-\mathrm{C} 1-\mathrm{C} 4$ & $114,5(3)$ \\
\hline $\mathrm{O} 2-\mathrm{B}-\mathrm{B}^{\prime}$ & $124,6(4)$ & $\mathrm{C} 3-\mathrm{C} 1-\mathrm{C} 4$ & $109,7(3)$ \\
\hline $\mathrm{B}-\mathrm{O} 1-\mathrm{C} 1$ & $111,6(3)$ & $\mathrm{O} 2-\mathrm{C}_{2}-\mathrm{C} 1$ & $102,2(2)$ \\
\hline $\mathrm{B}-\mathrm{O} 2-\mathrm{C} 2$ & $112,0(3)$ & $\mathrm{O} 2-\mathrm{C}_{2}-\mathrm{C} 5$ & $106,2(3)$ \\
\hline $\mathrm{O} 1-\mathrm{C} 1-\mathrm{C} 2$ & $103,1(2)$ & $\mathrm{O} 2-\mathrm{C} 2-\mathrm{C} 6$ & $107,1(3)$ \\
\hline $\mathrm{O} 1-\mathrm{C} 1-\mathrm{C} 3$ & $106,1(3)$ & $\mathrm{C} 1-\mathrm{C} 2-\mathrm{C} 5$ & $115,8(3)$ \\
\hline \multirow{2}{*}{$\mathrm{O} 1-\mathrm{C} 1-\mathrm{C} 4$} & $107,8(3)$ & $\mathrm{C} 1-\mathrm{C} 2-\mathrm{C} 6$ & $115,4(3)$ \\
\hline & & $\mathrm{C} 5-\mathrm{C} 2-\mathrm{C} 6$ & $109,1(3)$ \\
\hline
\end{tabular}

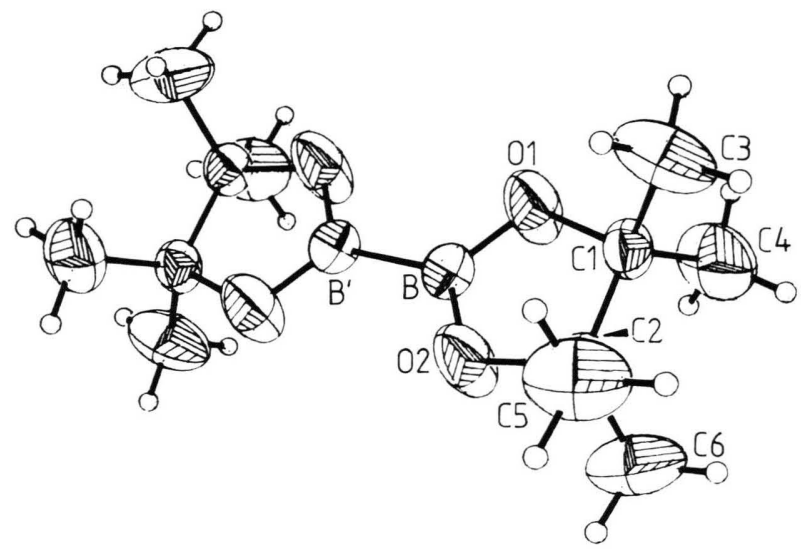

Abb. 1. Molekülstruktur von 5 in ORTEP-Darstellung.

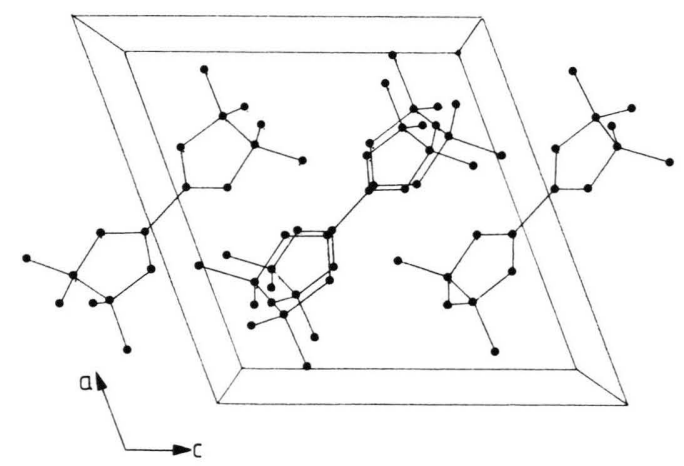

Abb. 2. Packung der 5-Moleküle in der Elementarzelle.
Kontakte (H4C-O2 282,2, H6B-O2 284,5 pm) bestimmt sind.

\section{Diskussion}

Die Molekülstruktur von 5 belegt, daß bei ihrer Synthese aus $\mathrm{B}_{2}\left(\mathrm{NMe}_{2}\right)_{4}$, Pinakol und $\mathrm{HCl}$ nicht das kondensierte Ringsystem 4 entsteht. Auch für das einfache Dioxaborolan-2-yl-dioxaborolan dürfte diese Struktur (3) vorliegen und nicht, wie aufgrund von kernresonanzspektroskopischen [7] und massenspektrometrischen Daten [6] angenommen wurde, das Isomere 4.

Daß die untersuchte Verbindung Struktur 5 besitzt, schlossen wir bereits aus der Lage seines ${ }^{11} \mathrm{~B}-\mathrm{NMR}$ Signals $\left(\delta^{11} \mathrm{~B} 33 \mathrm{ppm}\right)$ und seines ${ }^{17} \mathrm{O}-\mathrm{NMR}$-Signals $\left(\delta^{17} \mathrm{O} 161 \mathrm{ppm}\right)$ [9]. Die ${ }^{17} \mathrm{O}-$ Atome in Sechsringsystemen, etwa in 1,3,2-Dioxaborinanen mit einer $\mathrm{B}-\mathrm{O}-\mathrm{CMe}_{2}$-Atomfolge, sind nämlich deutlich besser als im Fünfring abgeschirmt [10]. Die Beobachtung, daß bei der massenspektrometrischen Fragmentierung von $\mathbf{3}$ praktisch kein DioxaborolanylKation-Fragment entsteht, während ein $(\mathrm{RO})_{2} \mathrm{~B}^{+}$Ion mit sehr hoher Intensität im Massenspektrum von $\mathrm{B}_{2}(\mathrm{OR})_{4}$ auftritt [6], ist leicht dadurch zu erklären, daß nur das Dialkoxybor-Kation 6 eine gute Resonanzstabilisierung erfahren kann, d.h. zwei unbesetzte $p$-Orbitale des Bors in die Bindung mit einbezieht, während bei 7 am Bor-Atom formal nur ein Elektronensextett vorliegt und damit keine optimale Stabilisierung erfolgt.

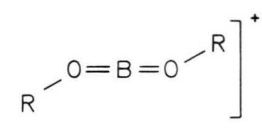

6<smiles></smiles>

7
5 kommt nahezu die Punktsymmetrie $\mathrm{D}_{2 \mathrm{~h}} \mathrm{zu}$. Neben $\mathrm{B}_{2} \mathrm{~F}_{4}$ [11] und $\mathrm{B}_{2} \mathrm{Cl}_{4}$ [12] besitzt es im festen Zustand eine (fast) planare $\mathrm{B}_{2} \mathrm{X}_{4}$-Struktur, im Gegensatz zu 1, dessen Diazaborolidin-Ringe um 61,6 bzw. $56,8^{\circ}$ gegeneinander verdrillt sind [5], da die $\mathrm{N}$ Methylgruppen aus sterischen Gründen keine Planarität zulassen.

Die B-B-Bindung in $\mathbf{5}$ ist etwas länger als in $\mathbf{1}$ und $\mathrm{B}_{2} \mathrm{~F}_{4}$ [11], aber kürzer als in $\mathrm{B}_{2} \mathrm{Cl}_{4}$ [12]. Somit zeichnet sich eine Substituentenabhängigkeit $a b$, die für $\mathrm{B}_{2} \mathrm{X}_{4}$-Verbindungen mit $\mathrm{X}=\mathrm{F}>\mathrm{N}>\mathrm{O}>\mathrm{Cl}$ zunimmt und möglicherweise darauf hinweist, daß mit Abnahme der BX-Bindungsordnung der B-B-Abstand zunimmt. Allerdings sind die XBX-Bindungs- 
winkel in den vier zum Vergleich herangezogenen Diboran(4)-Derivaten ungleich $\left(\mathrm{ClBCl} 120,5^{\circ}, \mathrm{FBF}\right.$ $115^{\circ}, \mathrm{NBN} 106,6^{\circ}, \mathrm{OBO} 111,1^{\circ}$ ), so daß in der B-BBindung unterschiedliche $p$-Anteile in der Bindung vorliegen sollten.

Die BO-Abstände in $\mathbf{5}$ sind gleich, im Vergleich mit anderen BO-Abständen vom DialkoxyboranTyp aber relativ kurz. Es ist jedoch bekannt, daß $\mathrm{BO}-\mathrm{Abstände}$ zu dreifach koordiniertem Bor beachtlich variieren können, wenngleich die meisten Abstände um $136 \pm 2$ pm liegen. Für Dioxaborolan-Systeme, wie sie in Kondensationsprodukten von Phenylborsäure mit Zuckern vorliegen, wurden Abstände von 137,6(6) [13], 139,4(3) [14] und 136,7 pm [15] röntgenographisch bestimmt. Für Dioxaborolan ergab die Strukturbestimmung mit Mikrowellen einen BO-Abstand von 136,8(10) pm [16]. Der vergleichsweise kurze BO-Abstand in $\mathbf{5}$ könnte somit auf einen größeren $\mathrm{BO}-\pi$-Bindungscharakter hinweisen, was mit dem $\delta^{17} \mathrm{O}$-Wert übereinstimmen würde.

Unter Berücksichtigung der erheblichen thermischen Anisotropie für die O-Atome könnte der tatsächliche Abstand bei Vorliegen eines stärker von der Planarität abweichenden Dioxaborolan-Ringes $\mathrm{zu}$ einem etwas größeren BO-Abstand führen. Erwähnt sei schließlich, daß die durchschnittliche BOBindungslänge in Tris(benzodioxaborolan-2-yl)amin, dessen Dioxaborolan-System planar ist, 138,1 pm beträgt [17].

Das Ergebnis der vorliegenden Untersuchung verdeutlicht, daß Verbindungen vom Typ $\mathbf{1}$ und $\mathbf{3}$ offensichtlich thermodynamisch stabiler als $\mathbf{2}$ und $\mathbf{4}$ sind. Verbindungen, in denen die Dibor-Einheit als Teil eines sechsgliedrigen Ringsystems auftritt (in Analogie zu 2 und 4), können jedoch als kinetisch kontrollierte Produkte NMR-spektroskopisch nachgewiesen werden [18]. Hierüber berichten wir an anderer Stelle.

\section{Experimenteller Teil}

Zur Röntgenstrukturanalyse stand ein SYNTEXP3-Vierkreisdiffraktometer zur Verfügung. NMRSpektren wurden mit einem JEOL-FX-90-Gerät registriert.

\subsubsection{5-Tetramethyl-1.3.2-dioxaborolan-1-yl-}

$4^{\prime} \cdot 4^{\prime} \cdot 5^{\prime} \cdot 5^{\prime}$-tetramethyl-1'.3'.2'-dioxaborolan (5)

Unter $\mathrm{N}_{2}$ tropfte man $\mathrm{zu}$ einer Lösung von $\mathrm{B}_{2}\left(\mathrm{NMe}_{2}\right)_{4}(19,8 \mathrm{~g}, 100 \mathrm{mmol})$ in $200 \mathrm{ml}$ Benzol unter Rühren Pinakol $(23,6 \mathrm{~g}, 200 \mathrm{mmol})$ in $150 \mathrm{ml}$ Benzol. $\mathrm{Zu}$ dieser Lösung fügte man sodann $150 \mathrm{ml}$ einer 2,7 M etherischen $\mathrm{HCl}$-Lösung. Nach 4-stdg. Rühren wurde das Unlösliche abgefrittet, das Benzol aus dem Filtrat i. Vak. entfernt und der Rückstand aus Pentan umkristallisiert. Ausbeute: 18,0 g (71\%), Schmp. $138^{\circ} \mathrm{C}$.

NMR-Daten (in $\mathrm{CDCl}_{3}$ ):

$\delta^{1} \mathrm{H}: \quad 1,036 \mathrm{ppm}$,

$\delta^{13} \mathrm{C}: 82,87 \mathrm{ppm}, \mathrm{C}, 24,51 \mathrm{CH}_{3},{ }^{1} J(\mathrm{CH}) 127 \pm 1 \mathrm{~Hz}$, $\delta^{11} \mathrm{~B}: 30,4 \mathrm{ppm}, \mathrm{h}(1 / 2)=445 \mathrm{~Hz}$.

$\mathrm{C}_{12} \mathrm{H}_{24} \mathrm{~B}_{2} \mathrm{O}_{4}(253,93)$

Ber. C 56,76 H 9,53,

Gef. C 56,20 H 9,49.

Für die Förderung unserer Arbeiten danken wir dem Fonds der Chemischen Industrie und der BASFAktiengesellschaft. Frau R. Wagner gilt Dank für Mithilfe bei der Durchführung dieser Arbeit.
[1] 152. Mitteil. W. Jackstieß, H. Nöth und W. Storch, Chem. Ber., im Druck.

[2] H. Nöth, P. Fritz und W. Meister, Angew. Chem. 73, 762 (1961).

[3] H. Nöth und P. Fritz, Z. Anorg. Allg. Chem. 324, 129 (1963).

[4] M. P. Brown, A. E. Dann, D. W. Hunt und H. B. Silver, J. Chem. Soc. 1962, 4648.

[5] H. Fußstetter, J. C. Huffman, H. Nöth und R. Schaeffer, Z. Naturforsch. 31b, 1441 (1976).

[6] G. L. Brubaker und S. G. Shore, Inorg. Chem. 8, 2804 (1969).

[7] G. N. Welch und S. G. Shore, Inorg. Chem. 7, 225 (1968).

[8] Positionen der H-Atome sowie weitere Angaben zur Röntgenstrukturbestimmung sind beim Fachinformationszentrum Energie, Physik, Mathematik in 7514 Eggenstein-Leopoldshafen unter Angabe der Autoren und des Zeitschriftenzitats unter CSD 50974 abzurufen.

[9] W. Biffar, H. Nöth, H. Pommerening und B. Wrackmeyer. Chem. Ber. 113, 333 (1980).
[10] B. Wrackmeyer und R. Köster, Chem. Ber. 115, 2022 (1982).

[11] L. Trefonas und W. N. Lipscomb, J. Chem. Phys. 28, 54 (1958).

[12] M. Atoji, P. J. Wheatley und W. N. Lipscomb, J. Chem. Phys. 27, 196 (1957).

[13] S. J. Rettig und J. Trotter, Can. J. Chem. 55, 3071 (1977).

[14] F. Zettler, H. D. Hausen und H. Hess, Acta Crystallogr. B 30, 1876 (1974)

[15] A. Gupta, A. Kiefel, G. Will und G. Wulff, Acta Crystallogr. B 33, 637 (1977).

[16] J. H. Hand und R. H. Schwendemann, J. Chem. Phys 45, 3349 (1966)

[17] G. J. Bullen und P. R. Mallinson, J. Chem. Soc. A $1970,2213$.

[18] H. Nöth und H. Pommerening, unveröffentlichte Versuche; vgl. H. Pommerening, Dissertation, Univ. München 1980 . 